\title{
Cystic duct anastomosis can be a viable option for biliary reconstruction in case of multiple ducts in right lobe living-donor liver transplantation
}

\author{
Aarathi Vijayashanker, Bhargava Ram Chikkala, Roshan Ghimire, Ravindra Nidoni, \\ Yuktansh Pandey, Rajesh Dey, Shaleen Agarwal, Subhash Gupta \\ Centre for Liver and Biliary Sciences, Max Super Speciality Hospital, New Delhi, India
}

\begin{abstract}
Backgrounds/Aims: Multiple ducts in right lobe living-donor liver transplant (LDLT) pose a technical challenge in biliary reconstruction. In the absence of separate recipient hepatic ducts for duct-to-duct anastomoses and certain demerits of hepaticojejunostomy, duct to duct anastomoses with the recipient cystic duct might be a possible solution.

Methods: A total of 329 recipients of LDLT who underwent two or more separate biliary anastomoses at our centre between January 2014 and November 2019 were studied retrospectively. Records of demographic data, donor and graft characteristics, operative details, postoperative biochemical parameters, and biliary complications were analysed.

Results: Of 329 recipients, 236 patients (71.7\%) underwent purely duct-to-duct (DD group) anastomoses, 38 patients (11.5\%) underwent at least one anastomosis with the cystic duct (CD group), and 55 patients (16.7\%) underwent at least one hepaticojejunostomy (HJ group). At one year, biliary complication rates of these three groups were $20.3 \%, 26.3 \%$, and $20.0 \%$, respectively $(p=0.68)$. Postoperative intensive care unit and overall hospital stay were similar among the three groups. Grades IIIa, IIIb, IV, and V Clavien-Dindo complications were identical. One-year patient survival and graft survival were also similar among the three groups.

Conclusions: Biliary outcomes using the cystic duct may have acceptable outcomes. Similar postoperative results as other means of biliary reconstruction could be anticipated with the cystic duct anastomoses in case of multiple ducts in the graft.
\end{abstract}

Key Words: Bile ducts, extrahepatic; Surgical anastomosis; Living donors; Liver transplantation; Complications

\section{INTRODUCTION}

Multiple ducts in right lobe living-donor liver transplant (LDLT) pose a technical challenge in biliary reconstruction. Although multiple ducts could be obtained by high hilar division of recipient bile duct, this may not be advisable in many situations. In cases of hepatocellular carcinoma, margins may

Received: February 22, 2021, Revised: May 15, 2021,

Accepted: May 17, 2021

Corresponding author: Aarathi Vijayashanker Centre for Liver and Biliary Sciences, Max Super Speciality Hospital, New Delhi 110017 , India

Tel: +91-7087390183, E-mail: aarthi.18@gmail.com

ORCID: https://orcid.org/0000-0003-0850-1101

Copyright (C) The Korean Association of Hepato-Biliary-Pancreatic Surgery

his is an Open Access article distributed under the terms of the Creative Commons Att BY NC bution Non-Commercial License (http://creativecommons.org/licenses/by-nc/4.0) which
permits unrestricted non-commercial use, distribution, and reproduction in any medium, provided the original work is properly cited. be compromised by division close to the porta. The most proximal part of bile duct might be ischemic as the vasculature is arising from below upwards. When ducts are close enough on the hilar plate or in a common sheath, a ductoplasty or duct-tosheath anastomoses using recipient common hepatic duct may be considered for reconstruction. However, when donor ducts are far apart, their anastomoses to right and left bile ducts might not be feasible always. In sick recipients, anastomosis to jejunum may also be fraught with complications such as risk of enteric leak and delay in initiation of enteral nutrition. It may also be a technical challenge due to thickened bowel from chronic oedema due to portal hypertension or portal vein thrombosis. In addition, anastomosis to proximal bile duct may introduce a "swan neck deformity", where the anastomosis is fine but the redundancy of the long bile duct creates an angulation, serving as a functional obstruction and making endoscopic retrograde cholangiography (ERC) difficult. In such situations, anastomosis to the cystic duct may be a solution. 
However, studies with large series on this subject have not been reported so far.

\section{MATERIALS AND METHODS}

This study was conducted at the Centre for Liver and Biliary Sciences, Max Super Speciality Hospital, Saket, New Delhi, India. Sample data were collected retrospectively from a prospectively created electronic database. Between January 2014 and November 2019, liver transplant recipients were identified using the following inclusion and exclusion criteria. This study conformed with the 1975 Declaration of Helsinki. It was approved by our ethical committee.

All cirrhotic patients with a model for end-stage liver disease (MELD) score more than 15 or unresectable, non-metastatic hepatocellular carcinoma were listed for LDLT at our centre. Donor work up and evaluation were done on an out- patient basis in four well-defined steps. Our protocol has been previously published at length. It has homogeneously remained the same since $2013[1,2]$. All cases were reviewed and discussed at weekly transplant meets before surgery.

Inclusion criteria:

1. Living donor liver transplant recipients

2. Right lobe grafts with two or more separate biliary anastomoses

Exclusion criteria:

1. Single biliary anastomoses, including ductoplasty, duct to sheath anastomoses, or isolated hepaticojejunostomy (HJ)

2. Left lobe grafts, paediatric transplants, patients of sclerosing cholangitis, common bile duct (CBD) stones

\section{Surgical reconstruction in case of multiple ducts}

Standard recipient hepatectomy was performed at our centre. The hepatoduodenal ligament was resected as high up into the parenchyma as feasible (except in cases of hepatocellular carcinomas or difficult recipient hepatectomies). After vascular reconstruction was completed, the redundant recipient bile duct was cut back to an appropriate length for a tension- free, ductto-duct anastomosis to the graft. Care was taken to confirm the viability of the ductal tissue at the level of anastomosis (fresh bleed at duct edge and good mucosal colour). The pericholedochal tissue containing the peribiliary vascular plexus was kept intact. The right hepatic artery was not dissected away from the bile duct, keeping the biliodigestive sheath intact. Sphincter of Oddi Dysfunction was ruled out by inserting an infant feeding tube down the bile duct into the duodenum. The arterial anastomosis of the donor artery was routinely performed to the recipient's left hepatic artery was routinely.

Our study population consisted of cases with at least two or more biliary anastomoses. Appropriate biliary reconstruction was performed for each case. Ductoplasty was not widely preferred at our centre due to concerns about recalcitrant strictures from previous experience. It was also difficult to perform when ducts were placed wide apart, a frequent occurrence in right lobe LDLT. Duct to duct anastomoses were done when two or more ductal ends (typically left and right hepatic ducts, rarely accessory duct orifices) were available on the recipient side. In case of a single orifice of recipient common hepatic duct, either a cystic duct anastomosis (after confirming size match and quality) or $\mathrm{HJ}$ was done for the other donor duct. This decision was taken intraoperatively. Recipients who were frail at the time of transplant, with previous abdominal surgeries and/or had oedematous bowel, inflammatory bowel disease, and abdominal cocoon were preferred to undergo a cystic duct anastomosis over HJ. The cystic duct lumen was checked for obstruction and straightened with a Watson-Cheyne probe. The duct was cut back until valves of Heister were no longer obstructive. The graft hepatic duct orifices were anastomosed to the recipient ducts in an end-to-end fashion using sequential interrupted, 7-0 polydioxanone stitches in a single layer, ensuring mucosal adherence. First, two corner sutures were taken at 3 o'clock and 9 o'clock position. The posterior layer stitches were tied with knots inside the lumen. The anterior layer was completed in a similar fashion, with knots outside. Hepaticojejunostomies were performed in interrupted fashion using a duct to mucosa technique, in similar fashion. Trans-anastomotic tubes were not used. Saline leak test and intraoperative cholangiogram were done in all cases by direct cannulation of the CBD and/or Roux loop. All reconstructions were performed by a single surgeon.

Graft vasculature and biliary system were assessed postoperatively using Doppler ultrasound. Standard triple drug-immunosuppressive regimen was used uniformly in our study cohort.

\section{Diagnosis of biliary complication}

Suspicious signs of bile leak included bilious drain output, fever or features of abdominal sepsis with/without biochemical indicators like hyperbilirubinemia and/or elevated alkaline phosphatase. Magnetic Resonance Imaging were performed for all suspicious cases. Magnetic resonance cholangiopancreatography (MRCP) was the imaging of choice for suspected biliary strictures.

\section{Management of biliary complication}

Persistent bile leaks required prolonged placement of surgical drains or postoperative placement of percutaneous catheters in bilious collections. Exploratory laparotomy and lavage were done if uncontrolled leaks were identified, causing intra-abdominal sepsis. Revision of biliary reconstruction was not attempted owing to frail portal tissue. Controlled leaks were managed with oral recycling of bile. If the leak was high in output or lasted more than six weeks, ERC and papillotomy were done, followed by stent insertion into the CBD.

Symptomatic biliary strictures identified on MRCP in the early post- transplant period (less than six weeks) were man- 
aged with percutaneous transhepatic biliary drainage (PTBD). PTBD internalization or ERC balloon dilatation and plastic stenting were done after six weeks of transplant to allow time for anastomotic healing. This procedure was repeated every three months. In case of persistent stricture, stents were exchanged every three months. While right anterior sectoral duct (RASD) strictures were preferably treated with ERC, right posterior sectoral duct (RPSD) strictures were managed with PTBD as endoscopic access of RPSD was often difficult due to the position of a regenerated allograft. When possible, dual stents (one in each duct) were placed to provide greater dilatation and prevent obstruction of the adjacent duct orifice due to the stent itself. Any failed ERC was managed with PTBD. All strictures involving $\mathrm{HJ}$ were managed with PTBD.

All biliary complications that required laparotomy or endobiliary/percutaneous transhepatic interventions were considered major complications. Complications that required percutaneous drainage of collection or prolonged surgical drain maintenance were considered minor complications.

\section{Statistical analysis}

Statistical analyses of averages and standard deviations were performed with Microsoft Excel, 2010 and IBM SPSS ver. 23.0 (IBM Corp., Armonk, NY, USA). Continuous variables were expressed as median and range. Categorical variables are expressed as number and percentages. Chi-square test was used to study the difference in categorical variables between groups. If the number was less than 5, then Fischer's exact test was used. Continuous variables were compared using Kruskal-Wallis test. If there was a significant difference, pairwise comparison was done using Mann Whitney test. Logistic regression was used to assess risk factors for biliary complication. Variables with $p<0.2$ were studied in a multivariate regression model. Their strength of association was expressed as odd's ratio/ $\operatorname{Exp}(B)$. All tests were two tailed and a $p$-value of less than 0.05 was considered statistically significant. Kaplan Meier method was used for survival function and significance assessed by log rank test.

\section{RESULTS}

A total of 329 recipients of LDLT who underwent two or more separate biliary anastomoses were identified. Of them, $236(71.7 \%)$ underwent purely duct-to-duct (DD Group) anastomoses, 38 (11.5\%) underwent at least one anastomosis with the cystic duct (CD group), and 55 (16.7\%) underwent at least one HJ (HJ group). Their demographic data, donor details, graft characteristics, and operative details are summarized in Table 1 . There was no significant difference in sex distribution $(p=0.55)$, median ages $(p=0.10)$, or body mass index $(p=0.59)$ of recipients among the three groups. ABO compatibility between donor and recipient $(p=0.51)$ and median MELD scores

Table 1. Recipient, donor, and perioperative characteristics of groups

\begin{tabular}{|c|c|c|c|c|}
\hline Characteristic & DD group $(n=236)$ & CD group $(n=38)$ & $\mathrm{HJ}$ group $(n=55)$ & $p$-value \\
\hline Recipient age (yr) & $51(21-63)$ & $50(26-66)$ & $45(15-66)$ & 0.10 \\
\hline Recipient sex (female) & $41(17.4)$ & $8(21.1)$ & $7(12.7)$ & 0.55 \\
\hline Recipient BMI $\left(\mathrm{kg} / \mathrm{m}^{2}\right)$ & $24(16-38)$ & $24.5(19-39)$ & $25(17.5-40)$ & 0.59 \\
\hline MELD score & $20(7-35)$ & $20(8-36)$ & $19(7-37)$ & 0.62 \\
\hline ABO compatibility & & & & 0.51 \\
\hline Identical & 143 & 23 & 37 & \\
\hline Compatible & 89 & 13 & 16 & \\
\hline Incompatible & 4 & 2 & 1 & \\
\hline Donor age (yr) & $31(18-45)$ & $32(18-50)$ & $32(18-59)$ & 0.91 \\
\hline Donor sex (female) & $71(30.1)$ & $13(34.2)$ & $17(30.9)$ & 0.87 \\
\hline Donor weight (kg) & $63(50-79)$ & $67(50-89)$ & $64(44-89)$ & 0.49 \\
\hline Graft weight (g) & $787(498-1,009)$ & 755 (379-990) & $738(486-940)$ & 0.71 \\
\hline GRWR & & & & 0.65 \\
\hline$<0.8$ & 42 & 6 & 7 & \\
\hline$\geq 0.8$ & 194 & 32 & 48 & \\
\hline Cold ischemia time (min) & $55(45-95)$ & $63(41-101)$ & $62(40-89)$ & 0.73 \\
\hline Warm ischemia time (min) & $31(20-50)$ & $32(22-54)$ & $32(19-54)$ & 0.63 \\
\hline Operative time (min) & $501 \pm 60$ & $530 \pm 65$ & $565 \pm 50$ & 0.04 \\
\hline No. of anastomosis & & & & 0.09 \\
\hline 2 & $201(85.2)$ & $27(71.1)$ & 46 (83.6) & \\
\hline 3 & $35(14.8)$ & 11 (28.9) & 9 (16.4) & \\
\hline
\end{tabular}

Values are presented as median (range), number (\%), number only, or mean \pm standard deviation.

DD group, underwent purely duct-to-duct; CD group, underwent at least one anastomosis with the cystic duct; HJ group, underwent at least one hepaticojejunostomy, BMI, body mass index; MELD, model for end-stage liver disease; GRWR, graft-to-recipient weight ratio. 
at the time of transplant were not significantly $(p=0.62)$ different either. Donor characteristics including age, gender, and body weight were also similar among the three groups $(p=0.91$, $p=0.87$, and $p=0.49$, respectively). There was no significant difference in graft weight, graft-to-recipient weight ratio, cold ischemia time (from the time of arterial ligation in the donor till the time the graft was out of ice), or warm ischemia time (from the time the graft was out of ice till portal reperfusion) either. However, the operative time was significantly longer in the HJ group $(p=0.04)$. All three groups were followed up for a minimum period of 12 months, with a median follow-up period of 35 months (12-72 months), 20 months (12-72 months), and 47.5 months (12-75 months), respectively.
Postoperative biochemical parameters of the study population including peak bilirubin and day-7 liver function tests were similar among the three groups (Table 2).

All patients were followed up for a minimum period of 12 months. At one year, biliary complication rate of the three groups were $20.3 \%, 26.3 \%$, and $20.0 \%$, respectively ( $p=0.68$ ). The overall rate of biliary complication was $27.3 \%(n=90)$ (Table 3). Bile leak rates in DD, CD, and $\mathrm{HJ}$ groups were $15.2 \%$, $21.1 \%$, and $14.5 \%$, respectively. Biliary stricture rates in these three groups were $12.7 \%, 13.2 \%$ and $5.4 \%$, respectively. There was no statistically significant difference in the incidence of biliary complications among the three groups (leaks, $p=$ 0.66 ; strictures, $p=0.29$ ) (Fig. 1). Overall, the stricture rate

Table 2. Postoperative trend of liver function tests

\begin{tabular}{|c|c|c|c|c|}
\hline Variable & DD group $(n=236)$ & CD Group $(n=38)$ & HJ group $(n=55)$ & Significance \\
\hline Peak bilirubin (mg/dL) & $8.0(1.91-20.8)$ & $8.3(1.93-23.8)$ & $7.9(1.95-34.8)$ & 0.12 \\
\hline Day 7 bilirubin (mg/dL) & $3.6(0.51-15.9)$ & $3.7(0.59-14.6)$ & $3.9(0.5-16.3)$ & 0.84 \\
\hline Day 7 ALT (IU/L) & 71 (266-399) & $72(272-448)$ & $70(5-335)$ & 0.79 \\
\hline Day 7 AST (IU/L) & $120(20-351)$ & $100(25-288)$ & $113(21-457)$ & 0.22 \\
\hline Day 7 ALP (IU/L) & $99(29-877)$ & 97 (30-995) & $100(21-998)$ & 0.21 \\
\hline Day 7 INR & $1.31(0.9-3.25)$ & $1.32(0.9-3.19)$ & $1.24(0.8-2.4)$ & 0.54 \\
\hline
\end{tabular}

Values are presented as median (range).

DD group, underwent purely duct-to-duct; CD group, underwent at least one anastomosis with the cystic duct; HJ group, underwent at least one hepaticojejunostomy; ALT, alanine transaminase; AST, aspartate transaminase; ALP, alkaline phosphatase; INR, international normalized ratio.

Table 3. Postoperative complications in both groups

\begin{tabular}{|c|c|c|c|c|}
\hline Complication & DD group $(n=236)$ & CD Group $(n=38)$ & HJ group $(n=55)$ & Significance \\
\hline Leaks & $36(15.2)$ & $8(21.1)$ & $9(16.4)$ & 0.66 \\
\hline Delayed stricture after leak & 11 & 5 & 2 & 0.16 \\
\hline Duration of stricture onset (mo) & 10 & 12 & 8 & 0.12 \\
\hline Strictures & $30(12.7)$ & $5(13.2)$ & $3(5.4)$ & 0.29 \\
\hline Duration of stricture onset (mo) & 12 & 8 & 6.5 & 0.10 \\
\hline \multicolumn{5}{|l|}{ Overall BC } \\
\hline Minor & $21(8.8)$ & $4(10.5)$ & $2(3.6)$ & 0.37 \\
\hline Major & $45(19.1)$ & $9(23.7)$ & $9(16.3)$ & 0.67 \\
\hline $\mathrm{BC}$ at 1-year & $48(20.3)$ & $10(26.3)$ & $11(20.0)$ & 0.68 \\
\hline Vascular complications & 1 & 1 & 2 & 0.10 \\
\hline Acute rejection & 6 & 2 & 2 & 0.63 \\
\hline Initiation of enteral nutrition (day) & 3 & 3 & 6 & 0.01 \\
\hline Postoperative ICU stay (days) & 8 & 8 & 8 & 0.10 \\
\hline Postoperative hospital stay (day) & $23(9-109)$ & $22(11-86)$ & $22(6-67)$ & 0.65 \\
\hline \multicolumn{5}{|l|}{ Clavien-Dindo } \\
\hline Grade IIla & 8 & 2 & 3 & 0.84 \\
\hline Grade IIIb & 4 & 1 & 1 & 0.92 \\
\hline Grade IV & 15 & 4 & 3 & 0.58 \\
\hline Grade V & 5 & 3 & 2 & 0.15 \\
\hline 1-year patient survival & $211(89.4)$ & $33(86.8)$ & $48(87.2)$ & 0.36 \\
\hline 1-year graft survival & $214(90.7)$ & $35(92.1)$ & $51(92.7)$ & 0.27 \\
\hline
\end{tabular}

Values are presented as number (\%), number only, or median (range).

DD group, underwent purely duct-to-duct; CD group, underwent at least one anastomosis with the cystic duct; $\mathrm{HJ}$ group, underwent at least one hepaticojejunostomy; BC, biliary complicationsl; ICU, intensive care unit. 
was higher in patients who developed postoperative leaks $(p=$ 0.002). Interestingly, not all leaks progressed to strictures. The majority $(66.0 \%)$ of leaks healed without further biliary morbidity.

In the DD group, 45 (19.1\%) patients required some form of surgical or endobiliary intervention. Of these patients, 4 (1.6\%) required laparotomy for uncontrolled bile leaks whereas $2(0.8 \%)$ required $\mathrm{HJ}$ for an intractable stricture. Twenty-two (9.3\%) patients benefitted from ERC while 5 (2.1\%) patients needed PTBD. Ten (4.2\%) patients required both ERC and PTBD. Minor biliary complications were seen in $21(8.8 \%)$ patients. In the CD group, 9 (23.7\%) patients developed major biliary complications. One (2.6\%) patient was re-explored for peritonitis and one patient (2.6\%) underwent HJ. Four (10.5\%)

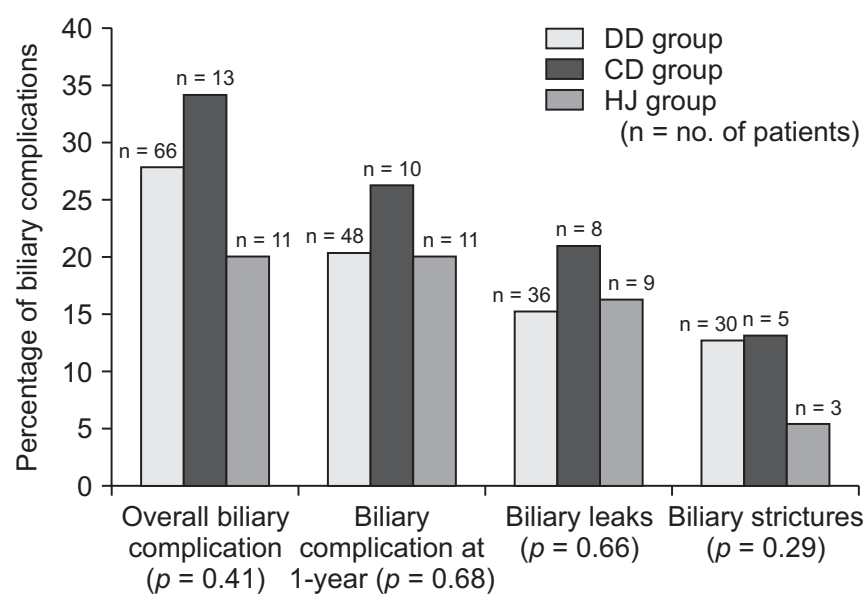

Fig. 1. Incidence of biliary complications in the study cohort. DD group, underwent purely duct-to-duct; $C D$ group, underwent at least one anastomosis with the cystic duct; $\mathrm{HJ}$ group, underwent at least one hepaticojejunostomy. patients responded to ERC whereas $4(10.5 \%)$ patients needed PTBD. One (2.6\%) patient required both ERC and PTBD. There was no significant difference in the amenability of ERC in patients with cystic duct anastomosis. PTBD use was similar across the two groups $(6.3 \%$ vs. $13.1 \%, p=0.17)$. In the $\mathrm{HJ}$ group, $9(16.3 \%)$ patients had major biliary complications and two $(3.6 \%)$ of them required laparotomy for $\mathrm{HJ}$ disruption. Five (9.1\%) patients required PTBD for management.

Hepatic artery thrombosis occurred in $0.4 \%(n=1), 2.6 \%$ $(\mathrm{n}=1)$, and $3.6 \%(\mathrm{n}=2)$ of patients in the three study groups, respectively $(p=0.1)$. There was no statistical difference in the incidence of acute rejection and steroid therapy among them $(p$ $=0.63$ ). Initiation of enteral nutrition was significantly delayed

Table 4. Univariate analysis of risk factors for biliary complications

\begin{tabular}{lccc}
\hline \multirow{2}{*}{ Risk factor } & \multicolumn{3}{c}{ Biliary complication } \\
\cline { 2 - 4 } & $p$-value & $\begin{array}{c}\text { Odds } \\
\text { ratio }\end{array}$ & $\begin{array}{c}\text { Confidence } \\
\text { interval }\end{array}$ \\
\hline Recipient age & 0.53 & 0.99 & $0.97-1.01$ \\
Sex (female) & 0.30 & 1.41 & $0.72-2.77$ \\
Recipient BMI & 0.68 & 0.91 & $0.90-1.07$ \\
Donor age & 0.005 & 1.03 & $1.01-1.06$ \\
Donor weight & 0.54 & 1.01 & $0.98-1.02$ \\
MELD score & 0.14 & 1.02 & $0.99-1.05$ \\
Cold ischemia time & 0.61 & 0.99 & $0.99-1.00$ \\
Warm ischemia time & 0.27 & 1.01 & $0.99-1.03$ \\
Graft weight & 0.81 & 1.00 & $0.99-1.00$ \\
GRWR & 0.56 & 0.77 & $0.33-1.83$ \\
Type of anastomosis & 0.30 & - & - \\
No. of ducts (two vs. more) & 0.86 & 1.06 & $0.53-2.14$ \\
\hline
\end{tabular}

BMI, body mass index; MELD, model for end-stage liver disease; GRWR graft-to-recipient weight ratio; -, not available.

Table 5. Univariate analysis for risk factors for bile leaks and strictures

\begin{tabular}{|c|c|c|c|c|c|c|}
\hline \multirow{2}{*}{ Risk Factor } & \multicolumn{3}{|c|}{ Biliary leaks } & \multicolumn{3}{|c|}{ Biliary strictures } \\
\hline & $p$-value & Odds ratio & Confidence interval & $p$-value & Odds ratio & Confidence interval \\
\hline Recipient age & 0.62 & 0.99 & $0.96-1.02$ & 0.85 & 0.99 & $0.97-1.03$ \\
\hline Sex (female) & 0.46 & 1.35 & $0.60-3.03$ & 0.46 & 0.69 & $0.25-1.86$ \\
\hline Recipient BMI & 0.58 & 0.77 & $0.67-2.68$ & 0.68 & 0.91 & $0.90-1.07$ \\
\hline Donor age & 0.01 & 1.03 & $1.01-1.06$ & 0.20 & 1.02 & 0.99-1.05 \\
\hline Donor weight & 0.40 & 1.01 & $0.98-1.03$ & 0.82 & 1.00 & $0.97-1.03$ \\
\hline MELD score & 0.36 & 1.02 & $0.98-1.05$ & 0.32 & 1.02 & $0.98-1.06$ \\
\hline Cold ischemia time & 0.21 & 0.99 & $0.98-1.0$ & 0.44 & 1.00 & $0.99-1.00$ \\
\hline Warm ischemia time & 0.01 & 1.03 & $1.01-1.06$ & 0.13 & 0.97 & $0.93-1.00$ \\
\hline Graft weight & 0.13 & 1.0 & $1.0-1.0$ & 0.33 & 0.99 & $0.99-1.00$ \\
\hline GRWR & 0.87 & 1.08 & $0.40-2.93$ & 0.45 & 0.62 & $0.17-2.18$ \\
\hline Type of anastomosis & 0.55 & - & - & 0.61 & - & - \\
\hline No. of ducts (two vs. more) & 0.19 & 1.72 & $0.81-3.65$ & 0.44 & 0.52 & $0.15-1.77$ \\
\hline
\end{tabular}

BMI, body mass index; MELD, model for end-stage liver disease; GRWR, graft-to-recipient weight ratio; -, not available. 
in the HJ group by three days $(p=0.01)$. Postoperative ICU and overall hospital stay were similar among the three groups. Grade IIIa, IIIb, IV, and V Clavien-Dindo complications were identical across the study groups. One-year patient survival and graft survival were not significantly different among the three groups either ( $p=0.36$ and $p=0.27$, respectively).

Finally, univariate, and multivariate analyses for risk factors of biliary complications were conducted. Donor age $(p=0.005$; OR, 1.03 ; 95\% CI, 1.01-1.06) was the only identifiable risk factor for overall occurrence of biliary complications on multivariate analysis. For bile leak, donor age $(p=0.01$; OR, 1.03; $95 \%$ CI, 1.01-1.06) and warm ischemia ( $p=0.01$; OR, $1.03 ; 95 \%$ CI, 1.01-1.06) were independent risk factors. No risk factor for biliary strictures was identified in our study cohort (Table 4, 5).

\section{DISCUSSION}

Biliary complications continue to plague LDLT as major causes of morbidity, with variable reports of incidence in literature ranging from $0.4 \%$ to $67 \%$ [3]. In addition to delaying recovery, biliary complications can significantly impact post-transplant quality of life and possibly reduce allograft survival [4]. Technique of reconstruction, smaller and multiple number of ducts, and hilar devascularization during dissection in the harvested right lobe grafts are considered as contributory factors.

The choice of biliary reconstruction in liver transplantation may be influenced by many factors, including the nature of liver disease (cholangiopathy vs. non-cholangiopathy aetiology), type of allograft or graft size (cadaveric or living donor), size of recipient and donor ducts, pre-existing biliary tract disease, abdominal trauma and/or surgery, intra-abdominal tuberculosis, and inflammatory bowel disease. During the initial years, Roux-en-Y HJ was the standard technique of reconstruction in adult LDLT based on reports of its use in the paediatric population [5]. With advancing surgical expertise, duct-to-duct anastomosis has been increasingly favoured globally with at least similar, if not better, outcomes as bilio-enteric drainage. Hepaticojejunostomies were found to have more bile leaks, which contributed to significant morbidity and mortality in the early postoperative period. There was a significant delay in the initiation of enteral nutrition in patients who underwent $\mathrm{HJ}$ in our study. On the other hand, biliary strictures were commonly encountered with duct-to-duct anastomoses, with a more delayed presentation frequently amenable to endoscopic or percutaneous curative therapy [6]. It is currently unclear which technique is superior to the other. Nevertheless, duct-toduct anastomosis is the preferred technique of biliary reconstruction in adult right lobe LDLT currently. Its merits include less operative time, less number of anastomoses, reduced postoperative ileus, preservation of physiological continuity of the tract, reduced enteric contamination of biliary tract due to intact Sphincter of Oddi and most importantly, and maintained endoscopic access to the bile duct $[7,8]$. However, HJ continues to remain the standard choice in left lateral segment or left lobe grafts.

Although it is never the first choice of reconstruction, when a reasonably sized cystic duct is present, it may be used to facilitate duct-to-duct anastomosis in certain circumstances when a Roux loop may be unfavourable, such as in cases of abdominal cocoon due to previous trauma and/or surgery, abdominal tuberculosis or inflammatory bowel disease, and bowel oedema. Evidence in the literature on outcome of cystic duct anastomoses in liver transplant are confined to case reports and case series [9-11]. We attempted to study the outcome of cystic duct vs. non-cystic duct anastomoses in adult right lobe LDLT performed at our centre.

The cystic duct has an average length of $2-4 \mathrm{~cm}$ and a calibre of 1-5 mm, with prominent endoluminal, spiral mucosal folds known as valves of Heister (named after its discoverer). Most commonly, the duct inserts laterally into the CBD in the mid portion. Extreme anatomical variations are frequently noted, which may include a short cystic duct, a medial insertion, a posterior or anterior insertion, a high or low insertion, a long parallel course with $\mathrm{CBD}$, and drainage into the right duct. Sometimes, there might be an aberrant right posterior sectoral duct drainage into the cystic duct [12].

While most cystic duct anomalies can be suitable for an anastomotic use, medial insertion, and long parallel course within a common fibrous tunnel with CBD may preclude its use. A previous cholecystectomy may also hamper the use of cystic duct. There is a theoretical possibility that the mucosal valves of the cystic duct may pose an obstacle to the passive bile flow from the right lobe as opposed to the intermittent, contractile flow from the gall bladder. Pina et al. [13] have studied the distribution and disposition of these valves through cadaveric dissection and found them to be present only in $69.5 \%$ of ducts. Moreover, they were present in full length in only $50 \%$ cases. At our centre, before performing biliary reconstruction, the cystic duct was always cut down closer to the CBD where the diameter of the duct was larger, and valves were nearly absent. This might have allowed us to bypass any possible obstruction. Chan and Fan [14], among many others, have noted that the recipient common hepatic duct may often be larger than the graft's right hepatic duct and should the ratio of size be greater than two, reduction of the former to a smaller size by plication is recommended. With the use of cystic duct, we believe that we may have inched closer to finding a size-matched ductal orifice to the second order bile ducts of the right lobe for reconstruction, producing comparable results to the use of hepatic ducts or HJs at one year of transplant $(p=0.68)$.

While studies are plenty in literature on the incidence of overall biliary complications in liver transplantation, there are limited studies that focus on multiple bile duct reconstruction. Therefore, it continues to be a matter of debate in LDLT [15]. Malagó et al. [16] have published a case series of five patients 
underwent end-to-side, duct-to-duct anastomoses to the CBD in case of multiple ducts in LDLT. While their results may appear promising, a long and vascularised bile duct was a pre-requisite for this technique. The pericholedochal sheath also may need to be dissected in the process to expose the bile duct for anastomosis, which may jeopardize the axial chain of blood vessels on the duct. Long-term results of this technique remain unclear. Salvalaggio et al. [17] have shown that the presence of multiple ducts is a risk factor for biliary complication in paediatric LDLT. Pamecha et al. [18] have identified the number of ducts and the number of anastomosis as independent predictors of biliary complications in adult LDLT. Noticeably, their multiple duct group also received $\mathrm{HJ}$ more frequently. On the contrary, DD was the most used technique in our study cohort $(n=274,83.2 \%)$. In addition, recipients requiring three biliary anastomoses (because of three or more ducts on the graft) were more likely to undergo a cystic duct biliary reconstruction over HJ in our study (28.9\% vs. $19.5 \%)$. Muhammad et al. [3] have conceded that multiple ducts have the same complication rate as single ducts in LDLT. More recently, Kollmann et al. [19] and Arikan et al. [20] have concluded that the number of biliary ducts and the type of anastomoses could not alter the outcome in LDLT. The BC rate of the former group at one year in multiple ducts was not significantly different from our findings $(20.0 \%$ vs. $20.9 \%, p=0.79)$. Nevertheless, a graft with one duct is favoured over a graft with multiple ducts by a surgeon for technical ease. There was no difference in the amenability of ERC in patients with cystic duct anastomosis. The use of percutaneous biliary intervention was not more in the CD group than in the DD group (6.3\% vs. $13.1 \%, p=0.17)$. From our experience, the valves of Heister have not proven to be more obstructive than a routine anastomotic stricture in the event of a biliary complication necessitating management. As expected, the HJ group was disadvantaged by a significantly delayed initiation of an enteral nutrition after transplantation and by the lack of ability to employ an endobiliary therapy in the event of biliary complications. Higher donor age correlated with increased overall biliary morbidity in our recipients, similar to findings of the Turkish group [20]. The one-year survival of the $\mathrm{CD}$ group and that of the overall cohort were similar to results of survival reported in literature $(86.8 \%, 88.7 \%$, and $89.0 \%$, respectively) [21].

Our study is limited by its retrospective nature, the small size of the cystic duct/HJ group, and single- centre data. Moreover, no more than one risk factor for biliary complication could be identified. This might be mainly because only perioperative factors were considered in our analysis, potentially excluding other indirect influencers. Nevertheless, the type of biliary anastomosis, the key question of the study, did not influence the outcome in our experience. Prospective, larger studies with longer postoperative follow up are needed to may provide more definitive evidences to ascertain the usefulness of cystic duct in biliary reconstruction.
Despite surgical advances, biliary complications continue to remain a significant problem in LDLT. Our experience suggests that biliary outcomes using the cystic duct may not be as poor as popularly speculated. If identified, complications could be successfully managed with an endoscopic or percutaneous therapy. Similar postoperative outcomes as other anastomoses may be anticipated in cystic duct with the use of a good surgical technique.

\section{ACKNOWLEDGEMENTS}

We would like to thank Mr. Vinod Karki and Mr. Vijaypal for their perennial support in collection and assembly of data.

\section{CONFLICT OF INTEREST}

No potential conflict of interest relevant to this article was reported.

\section{ORCID}

Aarathi Vijayashanker, https://orcid.org/0000-0003-0850-1101 Bhargava Ram Chikkala, https://orcid.org/0000-0002-3469-8190 Roshan Ghimire, https://orcid.org/0000-0002-9743-0458 Ravindra Nidoni, https://orcid.org/0000-0003-1246-469X Yuktansh Pandey, https://orcid.org/0000-0002-8864-9968 Rajesh Dey, https://orcid.org/0000-0002-0801-6205 Shaleen Agarwal, https://orcid.org/0000-0002-5187-7799 Subhash Gupta, https://orcid.org/0000-0002-7782-2593

\section{AUTHOR CONTRIBUTIONS}

Conceptualization: AV, SG. Data curation: AV, BRC, RG. Methodology: AV, SA, SG. Writing- original draft: AV, RN, RD, YP, SG. Writing- review \& editing: AV, BRC, RD, SA, SG.

\section{REFERENCES}

1. Vohra S, Goyal N, Gupta S. Preoperative CT evaluation of potential donors in living donor liver transplantation. Indian J Radiol Imaging 2014;24:350-359.

2. Rather SA, Nayeem MA, Agarwal S, Goyal N, Gupta S. Vascular complications in living donor liver transplantation at a high-volume center: evolving protocols and trends observed over 10 years. Liver Transpl 2017;23:457-464.

3. Muhammad ZM, Farah Z, Bushra Z, Tsan-S L, Chao-long C. Results of multiple biliary ducts anastomosis in living donor liver transplantation. Adv Res Gastroentero Hepatol 2016;2:555581.

4. Nguyen NTT, Harring TR, Goss JA, O’Mahony CA. Biliary reconstruction in pediatric liver transplantation: a case report of biliary complications and review of the literature. J Liver 2015;4:179.

5. Tanaka K, Uemoto S, Tokunaga Y, Fujita S, Sano K, Nishizawa T, et al. Surgical techniques and innovations in living related liver trans- 
plantation. Ann Surg 1993;217:82-91.

6. Kawachi S, Shimazu M, Wakabayashi G, Hoshino K, Tanabe M, Yoshida $\mathrm{M}$, et al. Biliary complications in adult living donor liver transplantation with duct-to-duct hepaticocholedochostomy or Roux-en-Y hepaticojejunostomy biliary reconstruction. Surgery 2002;132:48-56.

7. Rouch DA, Emond JC, Thistlethwaite JR Jr, Mayes JT, Broelsch CE. Choledochocholedochostomy without a T tube or internal stent in transplantation of the liver. Surg Gynecol Obstet 1990;170:239-244.

8. Liu CL, Lo CM, Chan SC, Fan ST. Safety of duct-to-duct biliary reconstruction in right-lobe live-donor liver transplantation without biliary drainage. Transplantation 2004;77:726-732.

9. Kadry Z, Cintorino D, Foglieni CS, Fung J. The pitfall of the cystic duct biliary anastomosis in right lobe living donor liver transplantation. Liver Transpl 2004;10:1549-1550.

10. Suh KS, Choi SH, Yi NJ, Kwon CH, Lee KU. Biliary reconstruction using the cystic duct in right lobe living donor liver transplantation. J Am Coll Surg 2004;199:661-664.

11. Asonuma K, Okajima H, Ueno M, Takeichi T, Zeledon Ramirez ME, Inomata Y. Feasibility of using the cystic duct for biliary reconstruction in right-lobe living donor liver transplantation. Liver Transpl 2005;11:1431-1434.

12. Turner MA, Fulcher AS. The cystic duct: normal anatomy and disease processes. Radiographics 2001;21:3-22.

13. Pina LN, Samoilovich F, Urrutia S, Rodríguez A, Alle L, Ferreres AR. Surgical considerations of the cystic duct and Heister valves. Surg J (N Y) 2015;1:e23-e27.

14. Chan SC, Fan ST. Biliary complications in liver transplantation.
Hepatol Int 2008;2:399-404.

15. Gondolesi GE, Varotti G, Florman SS, Muñoz L, Fishbein TM, Emre $\mathrm{SH}$, et al. Biliary complications in 96 consecutive right lobe living donor transplant recipients. Transplantation 2004;77:1842-1848.

16. Malagó M, Testa G, Hertl M, Lang H, Paul A, Frilling A, et al. Biliary reconstruction following right adult living donor liver transplantation end-to-end or end-to-side duct-to-duct anastomosis. Langenbecks Arch Surg 2002;387:37-44.

17. Salvalaggio PR, Whitington PF, Alonso EM, Superina RA. Presence of multiple bile ducts in the liver graft increases the incidence of biliary complications in pediatric liver transplantation. Liver Transpl 2005;11:161-166.

18. Pamecha V, Sasturkar SV, Sinha PK, Mohapatra N, Patil N. Biliary reconstruction in adult living donor liver transplantation: the allknots-outside technique. Liver Transpl 2021;27:525-535.

19. Kollmann D, Goldaracena N, Sapisochin G, Linares I, Selzner N, Hansen BE, et al. Living donor liver transplantation using selected grafts with 2 bile ducts compared with 1 bile duct does not impact patient outcome. Liver Transpl 2018;24:1512-1522.

20. Arikan T, Emek E, Bozkurt B, Mammadov E, Ceyhan O, Sahin T, et al. Does multiple bile duct anastomosis in living donor liver transplantation affect the postoperative biliary complications? Transplant Proc 2019;51:2473-2477.

21. Kim EJ, Lim S, Chu CW, Ryu JH, Yang K, Park YM, et al. Clinical impacts of donor types of living vs. deceased donors: predictors of oneyear mortality in patients with liver transplantation. J Korean Med Sci 2017;32:1258-1262. 